\title{
Socialización y radicalización política en militantes del Partido Comunista del Perú-Sendero Luminoso (PCP-SL)
}

\author{
Political socialization and radicalization in militants of \\ Communist Party of Peru-Sendero Luminoso (PCP-SL)
}

Eli Malvaceda / eli.malvaceda@usil.pe https://orcid.org/0000-0001-9506-4741

Universidad San Ignacio de Loyola, Perú

Juan Herrero / olaizola@uniovi.es

https://orcid.org/0000-0002-0146-2464

Universidad de Oviedo, España

Jossué Correa / jcorrea@umch.edu.pe

https://orcid.org/0000-0002-4166-7210

Universidad Marcelino Champagnat, Perú

\begin{abstract}
This article analyzes the process of socialization and political radicalization in militants of the Communist Party of Peru-Sendero Luminoso, who participated in the internal armed conflict in Peru between 1980 and 2000. Thus, through a phenomenological design, 16 in-depth interviews are performed, whose content analysis is carried out with support from the specialized software Atlas.ti 7.5. The results allow the identification of macro, meso and individual levels of socialization. Likewise, causal factors and catalyzing circumstances that influence and trigger radicalization are identified. It is concluded that there are not direct causes, but rather a progressive extremist escalation that crosses different levels of socialization, with emphasis on the meso and individual levels, and that exposed to catalyzing circumstances, either by rational or emotional way, leads to political radicalization.
\end{abstract}

Key words: socialization, political radicalization, causal factors, catalyzing circumstances, extremist escalation.

Resumen: Este artículo analiza el proceso de socialización y radicalización política en militantes del Partido Comunista del Perú-Sendero Luminoso, quienes participaron del conflicto armado interno en Perú en los años de 1980 a 2000. Así, mediante un diseño fenomenológico, se les realiza 16 entrevistas en profundidad, a las cuales se les efectúa un análisis de contenido con el soporte del software especializado Atlas.ti 7.5. Los resultados permiten identificar niveles de socialización macro, meso e individuales. Del mismo modo, se detectaron factores causales y circunstancias catalizadoras que influyen y desencadenan la radicalización. Se concluye que no existen causales directos, sino una escalada extremista que cruza por distintos niveles de socialización, con énfasis en los niveles meso e individual, y que expuesta a circunstancias catalizadoras sea por vía racional o emotiva conduce a la radicalización política.

Palabras clave: socialización, radicalización política, factores causales, circunstancias catalizadoras, escalada extremista. 


\section{Introducción}

La violencia política vivida en el Perú en el periodo de 1980 a 2000 fue la más cruenta de su historia. Tuvo como actores principales a las Fuerzas Armadas y al Partido Comunista del Perú- Sendero Luminoso (PCP-SL) (Comisión de la Verdad y Reconciliación [CVR], 2003a: 17). Según el Registro Único de Víctimas (Consejo de Reparaciones, 2012: 11), se reportaron 34,535 víctimas fatales; sin embargo, la cifra estimada de afectados ascendería aproximadamente a 69,280 (CVR, 2003b: 245). Este conflicto condujo a distintas secuelas a nivel sociopolítico, económico y psicosocial (CVR, 2003b: 133).

Entre 1995 y 2008 casi la totalidad de las investigaciones psicosociales sobre el conflicto armado se centraron en los afectados directos y familiares (Malvaceda, 2010: 193). En estos trabajos tiende a prestarse menos atención al "otro" implicado en el proceso, al perpetrador, a pesar de lo necesario de su abordaje (Theidon, 2004: 15; Cáceres, 2013: 6; Malvaceda, 2014: 3). En esta misma línea, como señalan diversos autores (Ferguson et al., 2015: 199), los estudios directos con estos actores son escasos.

Visto lo anterior, se desconocen aún los caminos y las raíces psicosociales que llevaron a personas comunes y corrientes al extremismo político. Así, en el presente estudio se busca conocer el proceso de socialización y radicalización política en los militantes del PCP-SL que participaron en el conflicto armado interno, entre 1980 y 2000.

\section{Socialización y radicalización política}

La socialización política es un proceso que se da a lo largo de toda la vida, está mediado por diversas agencias de la sociedad, a través de las cuales el individuo aprende las disposiciones de actitud y los patrones de conducta relevantes, desarrollando un marco de referencia que lo guía a plantearse opciones en torno a la política (Langton, 1969: 4). Tal proceso puede proporcionar la implicancia creciente de un individuo en causas radicales y ello conducir a acciones extremistas (Botha, 2014: 900). Como señalan Horgan y Taylor (2001: 18), la mayor parte de esta participación "se debe a la exposición gradual y la socialización, hacia un comportamiento extremo"; donde la radicalización es sólo una más de las vías para vincularse a él (Borum, 2011c: 3).

Se han realizado diversas revisiones sistemáticas en torno a la radicalización política (Wilner y Dubouloz, 2011 y 2010; Borum, 2011a y 2011b; Christmann, 2012; Kleinmann, 2012; Pisoiu, 2013; Hafez y Mullins, 2015; 
Klausen et al., 2016; Scarcella et al., 2016; McCauley y Moskalenko, 2017); empero, aún se dista de un consenso, pues la mayoría de los modelos no han sido sometidos a investigaciones exhaustivas (Borum, 2011b: 46) y además presentan deficiencias metodológicas (Kleinmann, 2012: 281).

Existe una diferencia entre la creencia radical (radicalismo) y la acción radical, tanto en una como en otra hay una diferencia de intensidad, ya sea desde una opinión neutral, hasta una obligación moral en el radicalismo; como desde una acción inerte hacia acciones radicales propiamente dichas (McCauley y Moskalenko, 2017: 211). Del mismo modo, se ha de tener en cuenta que existe tanto un radicalismo no violento, como uno violento; y este no necesariamente implica realizar actos extremistas (Pisoiu, 2013: 247). Asimismo, hay quienes cometen estos actos y no se encuentran radicalizados políticamente (Borum, 2011a: 8).

Como señalan Veldhuis y Staun (2009: 56 y ss.), la radicalización es un proceso psicosocial que conlleva cambios a nivel de las cogniciones, emociones y comportamientos dirigidos al aumento de la justificación y preparación para el conflicto intergrupal. Es un proceso individual, entendible sólo en su entorno social, que no sirve a un propósito claramente definido. Así, la radicalización demanda el compromiso personal, así como el sacrificio en defensa del endogrupo. Se diferencia de los caminos de acción, que se refieren al proceso de involucrarse en acciones extremistas (Borum, 2011a: 9). Diferentes investigaciones hacen hincapié en identificar y evidenciar la interdependencia de los niveles de socialización (macro, meso y microsociales) (Jordán, 2009: 198; McCauley y Moskalenko, 2017: 210), así como los factores causales (precondicionantes) y circunstancias catalizadoras (precipitantes) (Crenshaw, 1981: 381) que actúan a cada nivel y propician la radicalización.

A nivel macrosocial, se generan las condiciones previas de un entorno propicio para la radicalización. Un primer elemento es el contexto histórico. De acuerdo con Rénique (2015: 22), quien rastrea los orígenes de la radicalización política en el Perú, hasta inicios del siglo XX, fue en la década de 1960 cuando las agrupaciones radicales tomarían más forma, influidas por el foquismo guerrillero en América Latina, la "revolución militar" y la Reforma Agraria del gobierno de entonces, así como la emergencia de una nueva izquierda posguerrillera. La existencia y difusión de estas ideologías extremistas son una condición que favorece a la radicalización (Cliff y First, 2013: 293).

En la década de 1970 emerge el PCP-SL, deslindándose de sus antecesores, asumiendo el proceso de la "larga marcha” y volcándose al campesinado, 
realizando acciones de ideologización en la región andina (CVR, 2003c: 31; Degregori, 2011a: 163; Degregori, 2011b: 117; Rénique, 2015: 124). Sin embargo, no fue sino hasta 1980 cuando inició acciones armadas contra el Estado, las cuales se agudizaron en 1986 con la denominada "matanza de los penales”, donde ejecutaron a más de 200 senderistas (CVR, 2003d: 162), y que, a su vez, constituyó una circunstancia social precipitante, pues impulsó la adscripción al PCP-SL al explotar su imagen de víctimas y mártires (CVR, 2003e: 43). Tras ello, el conflicto escaló hasta la crisis extrema en 1992 cuando detuvieron al líder del PCP-SL, lo cual produjo que las acciones armadas se redujeran drásticamente hasta el 2000.

Aunado a lo anterior, el contexto social y político de la época evidencia un Estado débil, limitado en su ejercicio de control político; la República vacía y el Estado ajeno señalados por Lynch (2014: 69) dan muestra de la violencia estructural del país; ello matizado por el empobrecimiento e injusticia social dan razón en parte a aquellos sentimientos particulares de humillación, agravio o rebeldía, expresados en las grandes manifestaciones de docentes, padres y estudiantes a nivel nacional, las cuales posibilitan condiciones previas para el surgimiento de la radicalización (Horgan, 2005: 72; Jordán, 2009: 198; Wilner y Dubouloz, 2010: 38; Christmann, 2012: 26). A este respecto, los miembros del PCP-SL consideraron sus acciones como reacciones de defensa contra un enemigo (Degregori, 2011a: 106 y $2011 \mathrm{~b}$ : 144), tal explicación representa un sesgo de atribución de responsabilidad, que funciona en último término como un mecanismo de control para legitimar su conducta (Horgan, 2005: 41).

Ligado a lo anterior, los condicionantes culturales también resultan relevantes para el entendimiento de la radicalización (Arena y Arrigo, 2006: 8; Moyano, 2010: 74; Portocarrero, 2012: 15 y 2015: 107). El PCP-SL se inició como una organización eminentemente regional, constituida principalmente por profesores, estudiantes universitarios y maestros (Degregori, 2011b: 184; Rénique, 2015: 139), quienes conformaron la base social campesina para el partido, motivados por el poder de la idea, así como por la idea del poder (Degregori, 2011b: 188). Asimismo, la procedencia de esta masa crítica supuso la adopción de una concepción campesina tradicional de los roles familiares, donde el hombre representa y se hace cargo del espacio público, de la política, el orden; mientras que la mujer se encuentra relegada al espacio privado, lo íntimo, la solidaridad, el desprendimiento, el cuidado, así como al sufrimiento (Theidon, 2004: 73; Henríquez, 2006: 35, 36; Bracco, 2011: 1).

A nivel mesosocial o grupal se encuentran las fuentes directas de influencia, con quién se identifica, y a quién se compara el individuo. En este punto 
se localizan las redes del entorno social y político inmediato del individuo, tales como las redes de parentesco y amistad (Jordán, 2009: 208) que se pueden tejer en la familia, las instituciones educativas y la comunidad. Si bien tales redes resultan sumamente significativas, tampoco funcionan como determinantes en la radicalización política (Horgan, 2005: 54).

La familia constituida como un agente de socialización primaria es sumamente significativa en la socialización política; así es considerada por diversos estudios tanto en Perú (Degregori, 2011b: 186; Gavilan, 2013: 60), como en otros contextos internacionales (Florez-Morris, 2007: 626; Moyano, 2010: 95; Paul, 2010: 496; Christmann, 2012: 20; Acharya y Muldoon, 2015: 6; Hafez y Mullins, 2015: 964), puesto que la persona acepta el mundo, las representaciones sociales y la internalización de la cultura familiar (Mead, 1973: 200). Esta influencia es considerada como el primer paso en la transmisión de valores fundamentales (Botha, 2014: 897), y ayuda a definir y dotar de significado a los comportamientos apropiados e inapropiados (Morales et al., 2007: 436).

Por otra parte, las redes de amistad son de suma importancia en la socialización radical, pueden convertirse en la principal fuente de valores normativos, proporcionan recompensas emocionales, sentido de pertenencia, totalidad y significado (Jordán, 2009: 209; Kleinmann, 2012: 283). Para la formación de estas relaciones coinciden aspectos geográficos y de interdependencia funcional en actividades compartidas (Jordán, 2009: 210). En ese sentido, son las instituciones educativas y la comunidad donde se forman principalmente dichos lazos.

Diversas investigaciones nacionales (Ansión et al., 1993; CVR, 2003a; Lynch, 2006; Degregori, 2011a; Uccelli et al., 2013) e internacionales (Christmann 2012; Wilner y Dubouloz 2010; Hafez y Mullins 2015) señalan que la cercanía a grupos de pares radicalizados (como por ejemplo en las instituciones educativas) es un predictor de la radicalización (Veldhuis y Staun, 2009: 40), pues conllevan a la búsqueda de respuestas, iniciando la identificación con grupos radicales. Con base en lo anterior, las universidades también se constituyen como espacios de socialización política (Degregori, 2011b: 185). Aquí se da una mayor comprensión ideológica de los grupos radicalizados; a su vez, la presencia e influencia de estos es mucho mayor (Lynch, 1990: 45). En el Perú, tanto en los colegios como en las universidades existió una fuerte influencia de parte de los docentes como adoctrinadores y, a la vez, figuras de autoridad (Ansión et al., 1993: 142; Degregori, 2011b: 161). 
Aunado a esto, las comunidades radicalizadas, tanto las físicas como virtuales, las redes en torno a líderes, las redes sociales en prisiones (Asencios, 2013: 85) y la cercanía a grupos radicalizados son potenciales influencias para la radicalización política (Moghaddam, 2005: 163; Jordán, 2009: 211; King y Taylor, 2011: 609; Christmann, 2012: 15). Así, se generan procesos identitarios que implican aquellas decisiones ajustadas a la pertenencia y actuación colectiva a un determinado grupo. En el caso de grupos radicalizados, la militancia clandestina genera una identidad particular, lo cual crea relaciones más intensas, pues suponen lealtad, sacrificio e intimidad (Sageman, 2008: 68; Kleinmann, 2012: 284).

Estos grupos se constituyen como grupos primarios, lo cual, a su vez, genera una mayor cohesión grupal; además, la presencia de amigos y/o parientes en el grupo hace menos probable la traición; del mismo modo se exige la ruptura entre el individuo y aquellos que no forman parte de su nuevo grupo, generándose así grupos más aislados; por último, los elementos identitarios del grupo deshumanizan al enemigo, tornando más aceptable la violencia hacia él (Jordán, 2009: 208).

Finalmente, a nivel microsocial o individual se encuentran los factores psicosociales racionales, emocionales y normativos, tendientes a la radicalización política (Jordán, 2009: 201), los cuales sintetizan los niveles anteriores y han sido abordados por distintas investigaciones (Moyano, 2010; Botha, 2014; Veldhuis y Staun, 2009; McCauley y Moskalenko, 2017; Aly et al. 2016; Kleinmann, 2012; Horgan, 2005). Los aspectos racionales se entienden como las decisiones resultantes de un cálculo costo-beneficio y en correspondencia con una determinada estrategia. Entre los beneficios de pertenecer al grupo radical, está el de proporcionar un sentido de pertenencia al individuo, premiando el lazo personal, aumento del estatus, estado de ánimo y autoestima, al considerarlos héroes y glorias, respaldo de compañeros y familiares, así como aumento del sentido del riesgo, emoción y el peligro (Crenshaw, 1981: 385; Christmann, 2012: 27; Pisoiu, 2013: 252).

En cuanto a los aspectos emocionales, se considera a la actuación impulsada por las pasiones; en esta categoría se enmarcan los sentimientos como frustración, privación relativa, rabia ante la injusticia propia y de otros, deseos de venganza (Moghaddam, 2005: 162), entre otros. Eso es visible en los sucesos como la "matanza de los penales" (CVR, 2003e: 43). Al respecto, King y Taylor (2011: 610) afirman que el aspecto más importante en la decisión de radicalizarse es el componente afectivo, debido a las emociones provocadas por la injusticia vivenciada. 
Los aspectos normativos son aquellas decisiones ajustadas al marco valorativo del sujeto, el cual se identifica con los valores morales del grupo radicalizado y que, a su vez, dota de legitimidad moral a sus acciones (Wilner y Dubouloz, 2010: 34). A partir de ello identifica quiénes pertenecen al endo y al exogrupo; crea un marco impulsador de la radicalización, que tiene como base la conciencia y el sentimiento de injusticia, que recae sobre un colectivo al cual el individuo pertenece o al que es solidario; y finalmente, el convencimiento de que es posible vencer a la injusticia por medio de la movilización colectiva o insurrección (Ibarra y Letamendía, 1999: 395).

Las acciones extremistas se pueden organizar con la metáfora de la "escalera al terrorismo", propuesta por Moghaddam (2005), la cual ha de ser considerada en forma conjunta, puesto que ninguno de sus niveles es capaz de explicar por sí solo el proceso de radicalización. Asimismo, actúan en diferentes momentos y no necesariamente encadenados en un proceso ascendente (Wilner y Dubouloz, 2010: 45; Pisoiu, 2013: 246; Klausen et al., 2016: 70). Según el modelo, existiría en las personas una base de percepciones de injusticia y privación relativa, lo cual provocaría el deseo de un cambio político, económico, social o religioso. Tales aspectos se encuentran vinculados con la influencia histórica, social y cultural señalados anteriormente.

En la primera planta la persona exploraría sus opciones para luchar contra dicha situación; de no encontrar solución, es probable que la persona siga ascendiendo. En la segunda planta se desplaza la agresión hacia un grupo externo, como pueden ser las instituciones sociales en búsqueda de respuestas: personales o colectivas. En un tercer nivel la persona es persuadida a comprometerse con la moralidad de la organización, donde el endogrupo es moralmente justo, y el exogrupo se encuentra desvinculado moralmente. En este punto se justifican las acciones violentas; aquí se puede señalar la influencia de los grupos de pares radicalizadores (Cragin, 2014: 340). En el cuarto nivel la persona se sumerge en las relaciones sociales y las actividades del grupo, generando un pensamiento categórico y una legitimidad percibida de la organización extremista y sus metas, bajo la creencia de que los fines justifican los medios y polarizándose en relación con otros grupos. Asimismo, se plantean posibles recompensas. Finalmente, el quinto nivel es donde la persona desplaza los mecanismos inhibitorios necesarios para efectuar el daño a un tercero; aquí se dan dos subprocesos: la categorización social y la distancia psicológica, necesarios para efectuar actos extremos.

Aunado a los niveles de socialización, se tienen los factores causales y las circunstancias catalizadoras (Crenshaw, 1981: 381). Las primeras son las condiciones previas o predisponentes de la radicalización; son situaciones 
que no conducen forzosamente a la radicalización. Por su parte, las circunstancias catalizadoras son las precipitantes de la radicalización; se presentan como episodios significativos, como el inicio de las acciones armadas o de suma brutalidad policial, como las masacres u otras acciones críticas, las cuales pueden iniciar o reforzar los procesos de radicalización (Horgan, 2005: 73; Wilner y Dubouloz, 2011: 421; Klausen et al., 2016: 77). Cabe destacar que más que el énfasis en el momento crítico vivido, se ha de tomar en cuenta la representación de dicho momento para la persona, pues si bien pueden resultar fundamentales para la participación de unos, pueden no ser de importancia para otros (Borum, 2011b: 57).

Tal y como se ha mencionado, el proceso de radicalización política resulta vital para entender por qué las personas ejecutan acciones extremistas. $\mathrm{Si}$ bien los modelos presentan niveles y factores psicosociales que permiten explicar la radicalización, resulta pertinente explorarlos en función de la particularidad del fenómeno en el Perú, donde la literatura y la memoria oficial al respecto ha prestado generalmente una mayor atención al estudio de las víctimas, y una atención más limitada a los perpetradores. En este trabajo, se pretende, precisamente, estudiar el proceso de socialización y radicalización política en los militantes del PCP-SL que participaron en el mencionado conflicto armado interno.

\section{Metodología}

Se realizó una investigación con enfoque cualitativo, utilizando el diseño fenomenológico (Creswell, 2013: 42) que busca comprender la realidad desde el marco de referencia del sujeto que la experimenta. Para la construcción preliminar de la muestra, se plantearon los criterios del muestreo teórico (Strauss y Corbin, 2002: 219) y de representación socioestructural (Mejía, 2000: 166), procurando la representatividad y heterogeneidad de la muestra. Posteriormente, se usó el criterio del muestreo confirmatorio (Creswell, 2013: 93), para complementar los casos hasta alcanzar la saturación.

Se contó con 16 personas, militantes del PCP-SL, que participaron en el conflicto armado. Del total, 9 son hombres y 7 mujeres. El promedio de edad es 54 años. El 75\% cuenta con estudios universitarios; un porcentaje similar se encuentra recluido en un Establecimiento Penitenciario de la capital. El 56\% proviene de ciudades urbanizadas (Lima, Arequipa, Cusco y Callao). Asimismo, el promedio de edad a la cual se iniciaron en el conflicto armado fue 21 años. Finalmente, el $87 \%$ de los militantes se incorporó después de iniciadas las acciones armadas. 
En cuanto a la recolección de la información, la técnica utilizada fue la entrevista en profundidad semiestructurada. Para su construcción se partió del objetivo de la investigación en consonancia con el análisis teorético realizado (Strauss y Corbin, 2002: 45). La guía de entrevista fue validada mediante una entrevista piloto. Las entrevistas se llevaron a cabo entre octubre de 2015 y marzo de 2016.

Se consideraron aspectos éticos, como la obtención de los permisos necesarios de las autoridades correspondientes para realizar las entrevistas en los Establecimientos Penitenciarios de Perú. Además, se contó con el consentimiento informado de todos los participantes, el cual, por expresa petición, fue verbal. Se renombró a las entrevistas con un código, garantizándose así la confidencialidad de la información y la protección de los datos personales.

En cuanto al análisis de información, se hizo un análisis cualitativo de contenido temático, desde el enfoque del análisis crítico de discurso (Íñiguez, 2002: 93). Los datos se editaron siguiendo las convenciones de transcripción de Jefferson (1984: 368). El procedimiento de análisis se realizó mediante el software especializado de análisis de datos cualitativos Atlas.ti 7.5 y constó de tres etapas: 1) codificación a partir de las citas textuales de las entrevistas, posibilitando la codificación abierta, axial y selectiva (Strauss y Corbin, 2002: 110 y ss.); 2) estructuración de información a través de mapas semánticos presentando las megacategorías, categorías y subcategorías de sentido generadas; y 3) proceso de teorización, el cual consolida el diálogo teorético entre la teoría construida y la data empírica (Martínez, 2004: 278). Durante el proceso se llevó un sistema de memos analíticos teóricos y metodológicos (Strauss y Corbin, 2002: 250 y ss.).

Siguiendo a Milles et al. (2013: 277 y ss.), las tácticas consideradas para la construcción de significado fueron la frecuencia de las categorías (número de citas vinculadas con una categoría en particular; representa la fundamentación de la categoría) y la densidad teorética (número de vínculos que tiene una categoría con otras; indica la importancia explicativa de una categoría). Ambos aspectos representan la extensión (amplitud) y la comprensión (profundidad semántica) de los conceptos (Martínez, 2004: 294). Para el análisis de resultados, se han considerado significativas sólo aquellas categorías con alta frecuencia $(\geq 10)$ y densidad $(\geq 3)$ (colocados entre paréntesis tras cada categoría utilizada), descartando las que no cumplen con el criterio. 


\section{Resultados}

De acuerdo con el análisis (véase la Figura $1^{1}$ ), se identificaron cinco niveles de socialización ( 1 - 5), a partir de los cuales se desprenden factores causales (1.1 - 4.2) y psicosociales (1.1.1 - 4.2.2, 5.1 y 5.2), que influyeron en la radicalización política en militantes del PCP-SL.

A nivel macrosocial, los militantes del PCP-SL identifican que en su proceso de socialización política existió una profunda influencia social del contexto internacional, así como de los movimientos sociales (ya sea de docentes o estudiantiles) a nivel nacional (49-7), ello influyó en la normalización del discurso de revolución (19-6), así como en la percepción positiva hacia la denominada "lucha armada" (21-6). Ambos aspectos son altamente significativos en el entendimiento de la radicalización; se recrean entre sí, mostrando una relación dialéctica:

Todas esas situaciones formaron en mí mi visión, también mis lecturas, también la revolución de los partidos comunistas, la Unión Soviética, China, Cuba, entre otras. En ese tiempo se difundía y las lecturas estaban en función de eso. También en los partidos se sentía la necesidad de usar la lucha armada para la transformación del país (JCC, 55 años, 38: 38).

A nivel comunitario, resultan de suma relevancia la vivencia con clases sociales empobrecidas (36-4), lo cual les permitió desarrollar empatía y, consecuentemente, una mayor sensibilidad ante el sentimiento de injusticia para con ellos y otros en situación similar (32-7). Este último aspecto es muy importante, pues permite explicar otro tipo de influencias, como las individuales y familiares, lo cual se ve reflejado en lo siguiente:

Yo vengo de un hogar campesino, mis padres eran peones en una hacienda. Yo he visto de cerca la vida del campesino, la diferencia de clases en los años sesenta. Mientras los hacendados vivían de un modo, los campesinos tenían otro modo de vida... siempre hemos trabajado, los hijos de los peones tenían una educación hasta el tercer grado de primaria (IA, 54 años, 29: 29).

La influencia comunitaria afectó al nivel familiar sobre los principios morales, que conllevan una carga de cultura tradicional desarrollada en un entorno determinado (10-9). Si bien dicha categoría resultó poco fundamentada (escasa frecuencia), es a la vez sumamente explicativa (alta densidad) para entender la radicalización en los miembros del PCP-SL. A pesar de ello, los militantes del PCP-SL no identificaron referentes directos (11-6) en su participación, tal y como se señala a continuación: "En mi familia hay una vieja tradición militar, mi padre combatió en la guerra del 42 contra Ecuador,

1 Esta Figura se encuentra en el Anexo, al final del presente artículo (Nota del editor). 
mi hermano siguió en la fuerza aérea, entonces, mi madre era muy chauvinista, en mi familia había mucho esa tradición, la valentía" (AM, 52 años, 44: 44).

Los principios morales impartidos y la cultura tradicional generaron una influencia paterna en términos de política, orden y estudio (16-3); mientras que las mujeres señalaron tener una influencia maternal en términos de solidaridad (13-3), lo cual se asocia con el sentimiento de injusticia visto en el nivel comunitario. La influencia de otros miembros familiares (hermanos, pareja, hijos) no resultó significativa.

Mi formación ha sido familiar, de ser una persona solidaria, y si veo una injusticia, tratar de hacer algo para que eso cambie. La formación en sentimientos de ayuda a los demás y a la solidaridad es lo que cambia a uno. De mi madre aprendí la solidaridad. Mi padre y mis tíos fueron militares. Mi padre perteneció a la Marina. La disciplina, el orden, la preocupación por el estudio, fue por mi padre (VA, 55 años, 33: 33).

A nivel de las instituciones educativas, resultó altamente significativa la presencia de inquietudes sociales en los colegios, que conllevó a un perfilamiento identitario en los estudiantes (30-5); a su vez, esto se encontró asociado con la presencia de docentes significativos como figuras de autoridad (27-4). Ambos aspectos se encuentran influidos por las circunstancias del contexto nacional e internacional.

El profesor que nos entrenaba poco a poco iba hablando de política -él pertenecía a Patria roja-, así, un día le preguntamos: ¿cuántos grupos de izquierda existían? Y él nos nombró 16 grupos de izquierda, luego nos dijo que había uno más pero que era clandestino... yo con mi amigo, que también ha sido militante de Sendero decíamos: "Si es que no fuera una amenaza, entonces no estaría clandestino. Ahí nos dijimos: a ese busquémoslo" (AM, 52 años, 34: 36).

Por otra parte, la influencia universitaria resultó significativa en la medida que generó un ambiente político (21-5), el cual permitió el encuentro y participación en organizaciones políticas (25-4), así como en grupos de pares radicalizadores (14-6). Esto generó una sensibilización política en cuanto a los planteamientos radicales, y conllevó a una legitimidad y justificación de la violencia. Cabe mencionar que dichos aspectos fueron influidos por la normalización y percepción positiva de la lucha armada.

En la década de los ochenta y en universidad conocí al PCP, en ese tiempo yo me identifiqué con ellos, porque yo lo consideraba correcto. Cuando estábamos en clase yo me vinculé. Fuimos conversando, hasta que me invitaron a participar con ellos, ya desde ese entonces yo me interesaba por el debate. Debatíamos si era o no era correcta la lucha armada (JCC, 55 años, 51: 51).

Finalmente, a nivel individual, hay quienes señalan que su participación en la organización se debió a su autonomía moral (21-5), producto de un 
profundo proceso cognitivo. Así, explican que su decisión no se vio constreñida por otros agentes; al contrario, refieren que fue por razones personales.

Yo me inserto a participar del PCP-SL en la primera parte de la década de 1980. Lo puse todo en una balanza y dije: "Me incorporo", una decisión que pensé que era la mejor y decidí luchar por la transformación de la sociedad. Uno no podía ser un simple espectador. Fue con convicción, entregando la vida (VA, 55 años, 48: 48).

Por otro lado, hay quienes atribuyen las razones a desencadenantes externos (11-4), como lo fue el abuso de autoridad contra los miembros de dicha organización. Cabe recordar que ya las personas se encontraban sensibilizadas políticamente por la organización, situación que motivó su vinculación.

La experiencia del Frontón [matanza de los penales] fue la que más me motivó y conmovió, lo que ello hizo fue más hacerme avanzar, que retroceder, esto que me pasó a nivel personal, también se dio a nivel social, ya que muchas personas decidieron apoyar a partir de ese genocidio (GA, 49 años, 48: 48).

Los aspectos reseñados permiten graficar rutas en la socialización y posterior radicalización política seguida por los militantes del PCP-SL. Como se observa, no hay respuestas absolutas que la definan, tan sólo eslabones que, vinculados debidamente, desencadenan en la radicalización.

\section{Discusión}

En el presente estudio se plantea conocer cómo se da el proceso de socialización y radicalización política en los militantes del PCP-SL. Se analizan 16 entrevistas en profundidad con ellos. Los resultados permiten construir un marco de interpretación para entender tal fenómeno, el cual, lejos de ser monocausal, incorpora distintos caminos progresivos e interdependientes en el proceso de socialización. Asimismo, se identifican causales y catalizadores para la radicalización, los cuales obedecen a vías racionales, pasionales y normativas. Tales hallazgos se profundizan teniendo en consideración los aportes teóricos señalados con anterioridad (Borum, 2011b; Christmann, 2012; Jordán, 2009; Kleinmann, 2012; McCauley y Moskalenko, 2008; Veldhuis y Staun, 2009; Wilner y Dubouloz, 2010).

A nivel macrosocial, los resultados reafirman lo explicado por Botha (2014: 900), en cuanto a la existencia de una profunda influencia histórica, social, política y cultural en la socialización política, que generó las condiciones propicias para la radicalización. En efecto, las condiciones históricas (movimientos guerrilleros en Latinoamérica, Reforma Agraria, Revolución militar), sociopolíticas (República vacía, Estado ajeno, violencia estructural) y culturales (base social campesina, concepciones tradicionales de los 
roles familiares) condujeron a la normalización y a la percepción positiva del discurso de lucha armada (Moghaddam, 2005: 163). Ello es importante en el entendimiento de las condiciones previas de la radicalización; sin embargo, distan de detallar la influencia específica desde otros agentes (Botha, 2014: 897).

A un nivel mesosocial, se reconoce que la vivencia comunitaria con clases sociales empobrecidas condujo a construir representaciones sociales acerca de la situación de injusticia de las necesidades del otro (Arena y Arrigo, 2006: 161; Botha, 2014: 902). Esta localización social de identificación con un otro en desventaja les permitió a los futuros miembros del PCP-SL desarrollar empatía con los grupos radicalizados, creando las condiciones para una futura radicalización (Moghaddam, 2005: 163; Asencios, 2013: 85; Cáceres, 2013: 44).

Por otra parte, la familia, proveniente de aquella base social campesina, resalta en su importancia en torno a los principios impartidos, pues funda el límite entre lo correcto e incorrecto (Morales et al., 2007: 436; Paul, 2010: 496; Hafez y Mullins, 2015: 964). La incorporación de tales principios tiene lugar en las vivencias comunitarias, las cuales se encuentran influidas por la cultura andina y presentan, a su vez, una concepción tradicional de los roles familiares (Theidon, 2004: 73; Henríquez, 2006: 35 y 36).

Los militantes del PCP-SL presentaron una influencia paterna ligada a la política, el orden y el estudio; tal aspecto se condice con estudios internacionales (Arena y Arrigo, 2006: 161). En Perú, la influencia de las relaciones maternales sobre las militantes fue caracterizada por la solidaridad, el cuidado y el desprendimiento (Bracco, 2011:31 y ss.). En paralelo a ello, los resultados también indican ausencia de referentes directos que hayan conducido a la radicalización, lo cual es consistente con diversas investigaciones (Acharya y Muldoon, 2015; Botha, 2014; Florez-Morris, 2007; Horgan, 2005).

Los resultados permiten entender al PCP-SL como una estructura orgánica de raíces culturales profundamente tradicionales (Theidon, 2004: 73; Bracco, 2011: 2; Degregori, 2011b: 185; Portocarrero, 2012: 15 y 2015: 107). En ese sentido, permiten fundamentar que la influencia familiar sobre la radicalización se debe más a la representación de los roles familiares en un contexto cultural determinado, que a las personas en sí. La influencia familiar es importante mas no determinante, ya que posteriormente surgen otros significativos que ocupan su lugar, como el grupo de pares.

En los colegios se permite el planteamiento de inquietudes sociales, relacionadas tanto al contexto nacional e internacional, como a sus vivencias comunitarias concretizadas en el sentimiento de injusticia para con otros. Tal 
aspecto ha sido uno de los más recurrentes en los estudios sobre el PCP-SL (Lynch, 2006: 165 y ss.; Degregori, 2011b: 117). Ello es de suma relevancia, al permitir el perfilamiento político del estudiante (Degregori, 2011a: 163 y ss.). Un aspecto ligado a lo anterior es la presencia de docentes significativos, quienes representan figuras de autoridad y fuentes de adoctrinamiento, lo cual, a su vez, es respaldado por distintas investigaciones (Degregori, 2011b: 161; Uccelli et al., 2013: 29).

Por otro lado, la universidad se convirtió en un espacio de influencia por parte de las organizaciones políticas que la habitan (Lynch, 1990: 45; Ansión et al., 1993: 142). En concreto, los pares radicalizadores y los ideólogos presentes en la universidad generaron sensibilización política hacia las ideas radicales; esto permitió generar legitimidad y justificación de las acciones extremistas (Cragin, 2014: 340; Degregori, 2011b: 185). En la universidad se exploran distintas opciones para dar respuesta a las interrogantes de su realidad; así, los futuros militantes del PCP-SL tuvieron participación previa en organizaciones políticas (Lynch, 1990: 83). Ello estuvo influido por la percepción positiva hacia la lucha armada; sin embargo, no todos los que formaron creencias radicales desencadenaron en acciones de ese tipo (McCauley y Moskalenko, 2017: 206). Tal y como se puede apreciar, las vivencias políticas en los espacios educativos, más que los espacios en sí mismos, constituyeron un momento de alta significación para la asunción de una postura radical (Lynch, 1990: 45).

En esta etapa, las condiciones previas se perciben solubles, quedando a disposición del interesado dar respuesta a ello. Quienes a nivel individual deciden continuar con el desarrollo de una postura polarizada, elaboran discursos interpretativos que justifican y legitiman el uso de la violencia como recurso político (Arena y Arrigo, 2006: 42; Moyano, 2010: 100). Este punto es de vital importancia en la decisión de ser militante; de ahí que, a diferencia de los factores causales, cobren relevancia las circunstancias catalizadoras (Crenshaw, 1981: 381) que desencadenan la pertenencia al PCP-SL.

$\mathrm{Al}$ respecto, son dos las razones que señalan los miembros del PCP-SL en cuanto a su vinculación. Por un lado, quienes se iniciaron antes o al inicio de la lucha armada expresaron motivaciones vinculadas con una moral autónoma, no constreñidas por factores externos, sino por convencimiento propio. Se puede sugerir que este tipo de decisión fue principalmente cognitiva, ideológica, en función de una evaluación y estudio de su realidad. Dicha autonomía es discutible, pues hay múltiples influencias que conducen a la persona a tomar dicha decisión (Horgan, 2005: 72; Arena y Arrigo, 2006: 53). Ello no anula su agencia; al contrario, permite entenderla mejor desde sus determinantes. 
Otra de las explicaciones señaladas es la presencia de desencadenantes externos, como lo es el abuso de autoridad aplicada en contra del grupo con el cual se identifica la persona; esto es, el PCP-SL. Ello, influido a su vez por el sentimiento de injusticia para con los otros, generó solidaridad y una adscripción masiva al grupo en cuestión. A diferencia de la explicación anterior, en este caso las razones resultan ser sobre todo de corte afectivas. Vale recordar que tras la matanza de los penales se produjo el despliegue nacional de la violencia (CVR, 2003e: 43), ocasionando una mayor incorporación de militantes al PCP-SL. Esta explicación también es discutible en la medida que representa un sesgo de atribución de responsabilidad, que reduce la responsabilidad personal de los actos cometidos (Horgan, 2005: 41).

Si bien se indican dos determinantes en cuanto a la decisión de formar parte del PCP-SL -la vía racional y la emotiva-, no dejan de lado el aspecto normativo, el cual subyace a ambas, ya que por sobre las decisiones que se tomen, se encuentra el marco normativo del sujeto, adscrito al grupo de referencia.

\section{Conclusiones}

La socialización política que llevó a las personas a identificarse con el PCPSL se presentó como una escalada dinámica de radicalización. No existen factores determinantes; al contrario, se puede señalar un marco de referencia para la radicalización, que va desde los niveles macrosociales como generadores de las condiciones previas, en cuanto a la percepción positiva de la lucha armada y la normalización del discurso de revolución.

Posteriormente, el nivel mesosocial permite a nivel comunitario recoger el sentimiento de injusticia frente a las condiciones inadecuadas para con otros. La familia fue de suma importancia en cuanto a los principios de vida fomentados desde los roles paternos y maternos de la familia tradicional en la región andina.

En paralelo, desde las instituciones educativas, las inquietudes sociales, los docentes significativos (ideólogos), el ambiente político, los grupos de pares radicalizados y la participación en organizaciones políticas incrementaron dicha sensibilidad hacia los planteamientos radicales.

La investigación no proporciona evidencia acerca de personas significativas en la radicalización; sin embargo, indica que la influencia se debe a las representaciones de los roles asumidos por tales personas, sea en la comunidad, el hogar o las instancias educativas. 
Finalmente, a nivel microsocial, se plantean dos explicaciones en torno a la adhesión al PCP-SL. Por un lado, una vía racional, en función de una evaluación de la situación; y por otro, una vía emotiva que tuvo como catalizador una situación externa. Ambas se enmarcan dentro de las condiciones normativas, impuestas por el grupo y asumidas por el individuo.

Tal y como se puede apreciar, no existe un camino único hacia la radicalización; al contrario, hay una multiplicidad de caminos. Sin embargo, es pertinente considerar el planteamiento de niveles, factores causales y circunstancias catalizadoras para entender cómo las personas adoptan posturas radicales. Conocer esto es de vital importancia para continuar bregando en la lucha para que no se repita.

\section{Referencias}

Acharya, Khagendra y Muldoon, Orla (2015), "Why 'I' became a combatant: A study of memoirs written by Nepali Maoist combatants”, en Terrorism and Political Violence. Disponible en: http://dx.doi.org/10.1080/09546553.2015.1105797 [15 de junio de 2017].

Aly, Anne et al. (2016), "Introduction to the Special Issue: Terrorist Online Propaganda and Radicalization", en Studies in Conflict \& Terrorism, vol. 40, núm.1. Disponible en: http://dx.doi.org/10.1080/1057610X.2016.1157402 [12 de junio de 2017].

Ansion, Juan et al. (1993), La escuela en tiempos de guerra: Una mirada a la educación desde la crisis y la violencia, Perú: Tarea.

Arena, Michael y Arrigo, Bruce (2006), The terrorist identity. Explaining the terrorist threat, Estados Unidos: New York University Press.

Asencios, Dynnik (2013), Múltiples rostros, un solo sendero: aproximaciones a las motivaciones y militancia de jóvenes encarcelados de Sendero Luminoso en Lima, 1989-1992, tesis de Maestría, Perú: Pontificia Universidad Católica del Perú.

Borum, Randy (2011a), "Radicalization into Violent Extremism I: A Review of Social Science Theories”, en Journal of Strategic Security, vol. 4, núm. 4, Estados Unidos: Henley-Putnam University.

Borum, Randy (2011b), "Radicalization into Violent Extremism II: A Review of Conceptual Models and Empirical Research”, en Journal of Strategic Security, vol. 4, núm. 4, Estados Unidos: Henley-Putnam University

Borum, Randy (2011c), "Rethinking Radicalization”, en Journal of Strategic Security, vol. 4, núm. 4, Estados Unidos: Henley-Putnam University.

Botha, Anneli (2014), "Political Socialization and Terrorist Radicalization Among Individuals who Joined al-Shabaab in Kenya”, en Studies in Conflict \& Terrorism, vol. 37, núm. 11. Disponible en: http://dx.doi.org/10.1080/1057610X.2014.952511 [10 de junio de 2017].

Bracco, Diana (2011), Femineidad en mujeres que cumplen condena por el delito de terrorismo, tesis inédita, Perú: Pontificia Universidad Católica del Perú.

Cáceres, Cristina (2013), Discursos sobre reconciliación: el caso de los presos desvinculados de Sendero Luminoso y MRTA, tesis de Maestría, Perú: Pontificia Universidad Católica del Perú. 
Eli Malvaceda, Juan Herrero y Jossué Correa. Socialización y radicalización politica en militantes del Partido Comunista del Perú-Sendero Luminoso (PCP-SL)

Christmann, Kris (2012), Preventing religious radicalisation and violent extremism: $A$ systematic review of the research evidence, Reino Unido: Youth Justice Board.

Cliff, Christina y First, Andrew (2013), “Testing for Contagion/Diffusion of Terrorism in State Dyads”, en Studies in Conflict \& Terrorism, vol. 36, núm. 4. Disponible en: http:// dx.doi.org/10.1080/1057610X.2013.763599 [22 de junio de 2017].

CVR (Comisión de la Verdad y Reconciliación) (2003a), Informe Final, tomo I, Perú: CVR. CVR (Comisión de la Verdad y Reconciliación) (2003b), Informe Final, tomo II, Perú: CVR. CVR (Comisión de la Verdad y Reconciliación) (2003c), Informe Final, tomo IV, Perú: CVR. CVR (Comisión de la Verdad y Reconciliación) (2003d), Informe Final, tomo VII, Perú: CVR. CVR (Comisión de la Verdad y Reconciliación) (2003e), Informe Final, tomo III, Perú: CVR. Consejo de Reparaciones (2012), Sexto Informe Anual del Consejo de Reparaciones, Perú: Consejo de Reparaciones, Registro Único de Víctimas.

Cragin, Kim (2014), "Resisting Violent Extremism: A Conceptual Model for NonRadicalization”, en Terrorism and Political Violence, vol. 26, núm. 2. Disponible en: http:// www.tandfonline.com/doi/abs/10.1080/09546553.2012.714820 [9 de junio de 2017].

Crenshaw, Martha (1981), "The Causes of Terrorism”, en Comparative politics, vol. 13, núm. 4. Disponible en: http://www.jstor.org/stable/421717?origin=JSTOR-pdf [12 de junio de 2017].

Creswell, John (2013), Qualitative inquiry and research design: Choosing among five approaches, Estados Unidos: Sage.

Degregori, Carlos Iván (2011a), El surgimiento de Sendero Luminoso: Ayacucho, 1969-1979. Del movimiento por la gratuidad de la enseñanza al inicio de la Lucha Armada, Perú: Instituto de Estudios Peruanos.

Degregori, Carlos Iván (2011b), Qué difícil es ser Dios, Perú: Instituto de Estudios Peruanos. Ferguson, Neil et al. (2015), "Leaving Violence Behind: Disengaging from Politically Motivated Violence in Northern Ireland", en Political Psychology, vol. 36, núm. 2. Disponible en: http://onlinelibrary.wiley.com/woll/doi/10.1111/pops.12103/full [15 de junio de 2017].

Florez-Morris, Mauricio (2007), “Joining Guerrilla Groups in Colombia: Individual Motivations and Processes for Entering a Violent Organization”, en Studies in Conflict \& Terrorism, vol. 30, núm. 7. Disponible en: http://www.tandfonline.com/doi/ abs/10.1080/10576100701385958 [14 de junio de 2017].

Gavilan, Lurgio (2013), Memorias de un soldado desconocido. Autobiografía y antropología de la violencia, Perú: Instituto de Estudios Peruanos y Universidad Iberoamericana.

Hafez, Mohammed y Mullins, Creighton (2015), "The Radicalization Puzzle: A Theoretical Synthesis of Empirical Approaches to Homegrown Extremism”, en Studies in Conflict \& Terrorism, vol. 38, núm. 11. Disponible en: http://dx.doi.org/10.1080/105761 0X.2015.1051375 [17 de junio de 2017].

Henríquez, Narda (2006), Cuestiones de género y poder en el conflicto armado en el Perú, Perú: Consejo Nacional de Ciencia, Tecnología e Innovación.

Horgan, John (2005), The Psychology of terrorism, Estados Unidos: Routledge.

Horgan, John y Taylor, Max (2001), “The Making of a Terrorist”, en Jane's Intelligence Review, vol. 13, núm. 1, Estados Unidos: HIS Global.

Ibarra, Pedro y Letamendía, Francisco (1999), "Los movimientos sociales”, en Caminal, Miquel [ed.], Manual de Ciencia Politica, España: Tecnos. 
Convergencia Revista de Ciencias Sociales, núm. 78, 2018, Universidad Autónoma del Estado de México

Íñiguez, Lupicinio (2002), "El análisis del discurso en las ciencias sociales: variedades, tradiciones y práctica", en Íñiguez, Lupicinio [ed.], Análisis del discurso. Manual para las ciencias sociales, España: Universitat Oberta de Catalunya.

Jefferson, Gail (1984), "On the organization of laughter in talk about troubles", en Atkinson, Maxwell y Heritage, John [eds.], Structures of Social Action: Studies in Conversation Analysis, Estados Unidos: Cambridge University Press.

Jordán, Javier (2009), "Procesos de radicalizacion yihadista en España. Análisis sociopolítico en tres niveles", en Revista de Psicologia Social, vol. 24, núm. 2. Disponible en: http:// dx.doi.org/10.1174/021347409788041499 [13 de junio de 2017].

King, Michael y Taylor, Donald (2011), "The Radicalization of Homegrown Jihadists: A Review of Theoretical Models and Social Psychological Evidence", en Terrorism and Political Violence, vol. 23, núm. 4, Estados Unidos: Routledge Taylor \& Francis.

Klausen, Jytte et al. (2016), “Toward a Behavioral Model of 'Homegrown' Radicalization Trajectories", en Studies in Conflict \& Terrorism, vol. 39, núm. 1. Disponible en: http://www. tandfonline.com/doi/full/10.1080/1057610X.2015.1099995 [9 de junio de 2017].

Kleinmann, Scott (2012), "Radicalization of Homegrown Sunni Militants in the United States: Comparing Converts and Non-Converts", en Studies in Conflict \& Terrorism, vol. 35, núm. 4. Disponible en: http://dx.doi.org/10.1080/1057610X.2012.656299 [11 de junio de 2017].

Langton, Kenneth (1969), Political Socialisation, Inglaterra: Oxford University Press.

Lynch, Nicolas (1990), Los Jóvenes Rojos de San Marcos. El radicalismo universitario de los años setenta, Perú: El zorro de abajo.

Lynch, Nicolas (2006), Los últimos de la clase. Aliados, adversarios y enemigos de la reforma educativa en el Perú, Perú: Universidad Nacional Mayor de San Marcos.

Lynch, Nicolas (2014), Cholificación, república y democracia. El destino negado del Perú, Perú: Otra Mirada.

Malvaceda, Eli (2014), Alternativas pacificas ante la violencia politica desde los exmilitantes del PCP-SL, tesis de Maestría, Perú: Pontificia Universidad Católica del Perú.

Malvaceda, Eli (2010), Análisis cualitativo de estudios psicológicos sobre violencia politica, tesis inédita, Perú: Universidad Nacional Mayor de San Marcos.

Martínez, Miguel (2004), Ciencia y arte en la metodologia cualitativa, México: Trillas.

McCauley, Clark y Moskalenko, Sophia (2008), "Mechanisms of Political Radicalization: Pathways Toward Terrorism", en Terrorism and Political Violence, vol. 20, núm. 3. Disponible en: http://doi.apa.org/getdoi.cfm?doi=10.1037/amp0000062 [14 de junio de 2017].

McCauley, Clark y Moskalenko, Sophia (2017), "Understanding political radicalization: The two-pyramids model", en American Psychologist, vol. 72, núm. 3. Disponible en: http://doi.apa.org/getdoi.cfm?doi=10.1037/amp0000062 [17 de junio de 2017].

Mead, George (1973), Espiritu, Persona y Sociedad. Desde el punto de vista del conductismo social, España: Paidós.

Mejía, Julio (2000), "El muestreo en la investigación cualitativa”, en Investigaciones Sociales, vol. 4, núm. 5, Perú: Universidad Nacional Mayor de San Marcos.

Miles, Matthew et al. (2013), Qualitative Data Analysis: An Expanded Sourcebook, Estados Unidos: Sage.

Moghaddam, Fathali (2005), "The staircase to terrorism: A psychological exploration", en The American psychologist, vol. 60, núm. 2. Disponible en: http://www.ncbi.nlm.nih. gov/pubmed/15740448 [18 de junio de 2017]. 
Morales, José et al. [coords.] (2007), Psicología Social, España: Mc Graw Hill.

Moyano, Manuel (2010), Factores psicosociales contribuyentes a la radicalización islamista de jóvenes en España. Construcción de un instrumento de evaluación, tesis doctoral, España: Universidad de Granada.

Paul, Christopher (2010), "As a Fish Swims in the Sea: Relationships Between Factors Contributing to Support for Terrorist or Insurgent Groups, en Studies in Conflict \& Terrorism, vol. 33, núm. 6. Disponible en: http://dx.doi. org/10.1080/10576101003752630 [17 de junio de 2017].

Pisoiu, Daniela (2013), "Coming to Believe 'Truths' About Islamist Radicalization in Europe", en Terrorism and Political Violence, vol. 25, núm. 2. Disponible en: http://www. tandfonline.com/doi/abs/10.1080/09546553.2012.659361 [12 de junio de 2017].

Portocarrero, Gonzalo (2012), Profetas del odio. Raices culturales y lideres de Sendero Luminoso, Perú: Pontificia Universidad Católica del Perú.

Portocarrero, Gonzalo (2015), Razones de Sangre. Aproximaciones a la violencia politica, Perú: Pontificia Universidad Católica del Perú.

Rénique, José (2015), Incendiar la pradera. Un ensayo sobre la revolución en el Perú, Perú: La Siniestra ensayos.

Sageman, Marc (2008), Leaderless Jihad: Terror Networks in the Twenty-First Century, Estados Unidos: University of Pennsylvania Press.

Scarcella, Akimi; et al. (2016), "Terrorism, radicalisation, extremism, authoritarianism and fundamentalism: A systematic review of the quality and psychometric properties of assessments", en PLoS ONE, vol. 11, núm. 12. Disponible en: 10.1371/journal. pone.0166947 [ 14 de junio de 2017].

Strauss, Anselm y Corbin, Juliet (2002), Bases de la investigación cualitativa. Técnicas y procedimientos para desarrollar la teoria fundamentada, Colombia: Universidad de Antioquia.

Theidon, Kimberly (2004), Entre prójimos. El conflicto armado interno y la política de reconciliación en el Perú, Perú: Instituto de Estudios Peruanos.

Uccelli, Francesca et al. (2013), Secretos a voces. Memoria y educación en colegios públicos de Lima y Ayacucho, Perú: Instituto de Estudios Peruanos.

Veldhuis, Tinka y Staun, Jørgen (2009), Islamist radicalisation: a root cause model, Países Bajos: Netherlands Institute of International Relations Clingendael.

Wilner, Alex y Dubouloz, Claire-Jehanne (2010), "Homegrown terrorism and transformative learning: an interdisciplinary approach to understanding radicalization”, en Global Change, Peace \& Security, vol. 22, núm. 1. Disponible en: http://www.tandfonline. com/doi/full/10.1080/14781150903487956 [13 de junio de 2017].

Wilner, Alex y Dubouloz, Claire-Jehanne (2011), "Transformative Radicalization: Applying Learning Theory to Islamist Radicalization”, en Studies in Conflict \& Terrorism, vol. 34, núm. 5. Disponible en: http://dx.doi.org/10.1080/1057610X.2011.561472 [18 de junio de 2017]. 


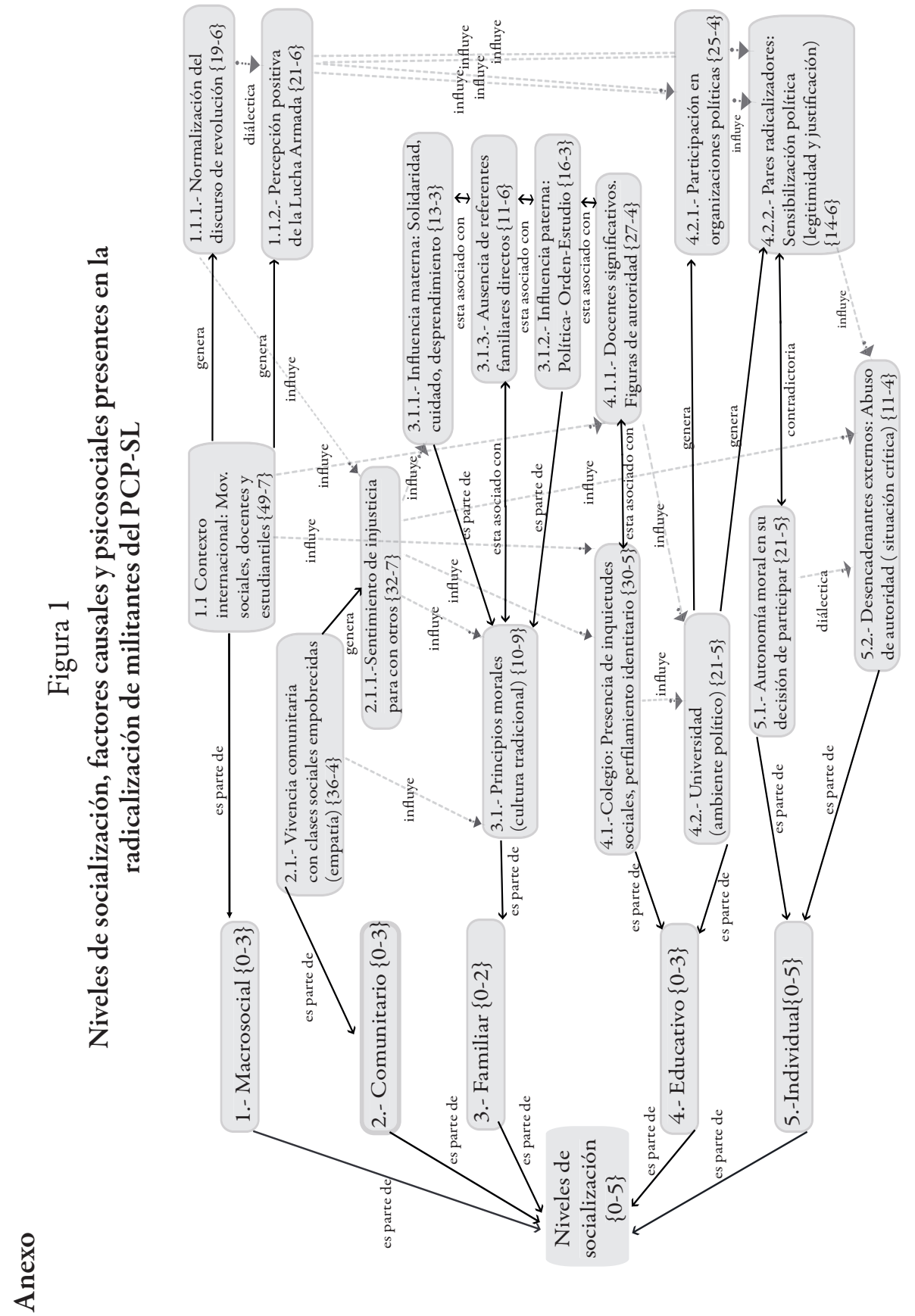

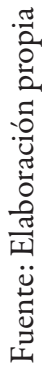


Eli Malvaceda. Magister en Psicología Comunitaria. Docente en el Departamento de Psicología de la Universidad San Ignacio de Loyola, Lima, Perú. Líneas de investigación: violencia social, conflicto armado, proyectos sociales y comunitarios. Publicaciones recientes: Manual de Ludotecas: Ludotecas comunitarias de Ventanilla, Perú: World Vision Peru (2013); Programa Cuenta cuento familiar: Experiencia Comunitaria, Perú: World Vision Peru (2013); "Análisis Psicosocial del sensacionalismo político en la prensa escrita: Elecciones municipales 2010", en Revista Peruana de Psicologia y Trabajo Social, vol. 1, núm. 1, Perú (2012).

Juan Herrero. Doctor en Psicología. Docente de la Facultad de Psicología de la Universidad de Oviedo, España. Líneas de investigación: psicología social de la violencia, violencia de pareja. Publicaciones recientes: Herrero, Juan; Rodríguez, Francisco y Torres, Andrea, "Acceptability of Partner Violence in 51 Societies", en Violence Against Women, vol. 23, núm. 3, USA (2017); Herrero, Juan; Rodríguez, Francisco; Torres, Andrea y Juarros, Joel, Intimate partner violence against women in the European Union: The influence of male partners' traditional gender roles and general violence, en Psychology of Violence, vol. 7, núm. 3, USA (2017); Rodríguez, Francisco; Herrero, Juan; Franco, Luis; Bringas, Carolina; Paino, Susana y Pérez, Beatriz, "Validation of Dating Violence Questionnarie-R (DVQ-R)", en International Journal of Clinical and Health Psychology, vol. 17, núm. 1, España (2017).

Jossué Correa. Magister en Bioestadística. Docente de la escuela de Psicología de la Universidad Marcelino Champagnat, Lima, Perú. Líneas de investigación: psicometría, violencia familiar.

Recepción: 1 de septiembre de 2017.

Aprobación: 31 de mayo de 2018. 
